medical journals reveals 396 medical reversals. eLife 8: e45183. doi: 10.7554/eLife.45183.

Macklin, R., and C. Natanson. 2020. Misrepresenting "usual care" in research: An ethical and scientific error. The American Journal of Bioethics. 20(1): 31-39.

Schmidt, B., R. K. Whyte, E. V. Asztalos, et al. 2013. Effects of targeting higher vs lower arterial oxygen saturations on death or disability in extremely preterm infants: A randomized clinical trial. JAMA 309(20): 2111-2120. doi: 10.1001/jama.2013.5555.
SUPPORT Study Group of the Eunice Kennedy Shriver NICHD Neonatal Research Network, Carlo, W. A., N. N. Finer, et al. 2010. Target ranges of oxygen saturation in extremely preterm infants. The New England Journal of Medicine 362(21): 1959-1969.

The BOOST II United Kingdom, Australia, and New Zealand Collaborative Groups. 2013. Oxygen saturation and outcomes in preterm infants. The New England Journal of Medicine 368(22): 2094-2104.

\title{
What Research Ethics (Often) Gets Wrong about Minimal Risk
}

\author{
Patrick Bodilly Kane ${ }^{a}$ (D), Scott Y. H. Kim ${ }^{b}$, and Jonathan Kimmelman ${ }^{a}$ \\ ${ }^{\mathrm{a}}$ McGill University; ${ }^{\mathrm{b}}$ National Institutes of Health
}

Trials that randomize patients to different "usual care" treatments play a crucial role in helping healthcare systems optimize the delivery of care. Yet, as illustrated by controversies surrounding clinical trials such as ARMA (The Acute Respiratory Distress System Network et al. 2000), TRICC (Hébert et al. 1999), and SUPPORT (SUPPORT Study Group of the Eunice Kennedy Shriver NICHD Neonatal Research Network 2010), their conduct is often met by controversy. Macklin and Natanson identify one often neglected aspect of the debates: ambiguity in the definition of "usual care." Designing informative trials often presses investigators to standardize treatments in ways that may depart from typical practice-for example, extremizing them. This, in Macklin and Natanson's view, exposes patients to risks and uncertainties they would otherwise not encounter in care.

But another controversy looms over usual care trials-indeed, it looms over whole swaths of research activities, including quality improvement: the definition of minimal risk. Many commentators argue that trials that randomize patients to treatments they might otherwise receive in practice constitute no risk trials for patients (assuming they deploy no procedures that serve a purely research purpose, like medically unnecessary biopsies). This view has many practical implications for the research enterprise since it opens up the prospect of waiving informed consent and expediting
IRB review. We think the view that randomization to standard treatments always presents zero risk rests on four misunderstandings about the nature and ethical properties of risk in human research.

The first is a misunderstanding of the morally appropriate reference class. A "reference class" is a set of events that are used to define risk. Those who argue that randomization presents no risk seem to base this assessment on a population-based reference class. They rightly point out that it is unknown which treatment-if any-will prove superior. This means that, in advance of the trial, there are no grounds for believing the reference class of patients entering a trial will be worse off on average than the same population forgoing the trial. So if patients in one arm do worse than the other, their welfare losses will be offset by the welfare gains of patients in the other arm. The problem is that research ethics explicitly rejects the notion that aggregate welfare can run roughshod over the welfare of individual patients. Instead, it defines the reference class at the level of the individual patient. If our reference class is the individual patient's risk of harm, the question we have to ask is whether there is a meaningful probability that some individuals will be made worse off by participating in the trial.

The second misunderstanding concerns the counterfactual nature of risk. By definition, risks associated with an activity are measured against the counterfactual 
of what would happen if the activity were not pursued. For a traveler the risks of flight are measured against the counterfactual of some other action, like traveling the same distance by rail. The risks of trial participation for an individual are logically measured against what an individual's care trajectory would have been had they pursued care outside of a trial. Randomization potentially abrogates that care trajectory. Considered with our first point the question of whether usual care trials present risk boils down to whether randomization might lead to worse outcomes for some patients.

The third misunderstanding is a conflation of randomness with arbitrariness (Lantos et al. 2015). Defenders of the "no risk" view argue that randomization is consistent with receiving care since it is a matter of chance which standard of care a patient receives in a practice setting. This reasoning has several problems. First, a clinic's selection of treatment is at best underdetermined by evidence and at worst arbitrary; it is not random. Indeed, were it truly random, observational studies would largely supplant clinical trials. Human factors-like geography, history, training, reputation, or religious affiliation shape whether a patient receives one treatment or another. Randomization negates the expression of those preferences in order to isolate the causative effects of a treatment. On top of this, randomization fixes allocation ratios-typically at 1:1while community practices rarely split evenly like this (Chen and Kim 2016). For example, where 80\% community practices might use one treatment and the remaining $20 \%$ the other, a trial would typically expose an excess of $30 \%$ of individuals to a treatment that might be inferior. Thus, it cannot even be said at the aggregate level that randomization produces the same result as assignment to treatment in clinical practice.

The fourth misunderstanding is how risk relates to clinical equipoise. In order for any randomized trial to be ethically conducted, it must be in clinical equipoise; that is, there must be uncertainty in the medical community about which treatment is most effective and this uncertainty must be substantial enough to warrant study. This uncertainty means randomization might redirect a patient towards a better performing treatment. It also might do the reverse. Thus in an ethical trial, there must be some genuine possibility that a patient is made worse off by participating, which is the very definition of a risk to a patient. Indeed, if one of the treatments is, in fact, superior and the participants include both those who would have received one standard of care and those who would have received the other, then there is a guarantee that some trial participants will be made worse off.
Many standard treatments diffuse into clinical practice without good evidence they are effective and/or more effective than other standard treatments. This under-determination of safety and efficacy harms patients and healthcare systems. Classifying usual care trials as minimal risk is appealing in that it removes one major barrier to addressing such ubiquitous shortfalls of evidence. However, as we have outlined above, the fact that in usual care trials no patient is knowingly deprived standard of care, does not entail that usual care trials are necessarily minimal risk.

We close by addressing some implications of the above analysis. First, our argument is relevant for informed consent. Informed consent exists in part because of the necessity to honor enrollment preferences of prospective research subjects based on the potential risk and benefits of the trial. As we have argued, enrollment in a usual care clinical trial opens the possibility of a patient being made worse off than they would otherwise have been had they not participated in the trial, and this is exactly the kind of information that must be presented to a patient in informed consent of any kind. In this regard, it is important to emphasize that even if there is equipoise (i.e. there is no consensus that patients will be made better or worse off by enrolling), research subjects can have legitimate grounds for declining enrollment that are rooted in preferences that autonomy ought to honor. As one example: a risk averse individual will generally avoid situations that present the possibility of leaving them worse off. In certain circumstances risk averse patients would be less inclined to enter trials involving randomization to usual care. Clearly, research ethics needs to get a handle on this.

Second, we believe that the above analysis explains why a complete waiver of consent would be inappropriate for most usual care trials. But we also believe that regulations need to be written in a manner that allows alterations (but not waivers) of informed consent for some usual care trials even where research risk is above minimal. Or, it may be that consent processes for usual care trials can be streamlined more than current practice assumes. We suspect that most of the goals of usual care trials could be met with these measures but there is insufficient space in this essay to further expound on these possibilities.

Last, we do not deny that there are some usual care randomized trials that present minimal or no risk. Nevertheless, in general, where usual care trials are aimed at measuring outcomes that entail large gains or losses of welfare, they cannot generally be presented as minimal risk. 


\section{DISCLOSURE STATEMENT}

The authors declare no competing interests of relevance to the present subject matter. The opinions expressed by SK are his own and do not represent the views of the NIH, DHHS, or the US government.

\section{FUNDING}

This work was supported by the Canadian Institutes of Health Research [EOG 131588].

\section{ORCID}

Patrick Bodilly Kane (D) http://orcid.org/0000-00031050-570X

Jonathan Kimmelman (iD http://orcid.org/0000-00031614-6779

\section{REFERENCES}

Chen, S. C., and S. Y. Kim. 2016. A framework for analysis of research risks and benefits to participants in standard of care pragmatic clinical trials. Clinical Trials: Journal of the Society for Clinical Trials 13(6): 605-611. doi: 10. $1177 / 1740774516656945$.

Hébert, P. C., G. Wells, M. A. Blajchman, et al. 1999. Transfusion requirements in critical care investigators for the Canadian critical care trials group. A multicenter, randomized, controlled clinical trial of transfusion requirements in critical care. The New England Journal of Medicine 340(6): 409-417. doi: 10.1056/NEJM199902113400601.

Lantos, J. D., D. Wendler, E. Septimus, S. Wahba, R. Madigan, and G. Bliss. 2015. Considerations in the evaluation and determination of minimal risk in pragmatic clinical trials. Clinical Trials: Journal of the Society for Clinical Trials 12(5): 485-493. doi: 10.1177/1740774515597687.

SUPPORT Study Group of the Eunice Kennedy Shriver NICHD Neonatal Research Network. 2010. Target ranges of oxygen saturation in extremely preterm infants. The New England Journal of Medicine 362(21): 1959-1969.

The Acute Respiratory Distress Syndrome Network, R. G. Brower, M. A. Matthay, et al. 2000. Ventilation with lower tidal volumes as compared with traditional tidal volumes for acute lung injury and the acute respiratory distress syndrome. The New England Journal of Medicine 342(18): 1301-1308.

\title{
"Unusual Care": Groupthink and Willful Blindness in the SUPPORT Study
}

\author{
George J. Annas ${ }^{a}$ and Catherine L. Annas ${ }^{b}$ \\ ${ }^{\mathrm{a} B o s t o n}$ University School of Public Health; ${ }^{\mathrm{b}}$ Beth Israel Deaconess Medical Center
}

The SUPPORT study of extremely premature newborns seems likely to go down as one of the most controversial studies of the 21st century (SUPPORT Study Group 2010). We previously suggested that the researchers in SUPPORT were "legally blind" in failing to understand that the "standard" that defines the content of informed consent is set by law, including the federal regulations, not by what physicians "usually" do or don't do (Annas and Annas 2013). Macklin and Natanson, also early critics of the SUPPORT study's failure to disclose the increased risk of death posed by the study, (Macklin et al. 2013) attack the study's methodology itself in this issue, arguing that even on its own terms SUPPORT was fatally flawed (Macklin and Natanson 2020). Specifically, they argue that one arm of the study (the low oxygen arm) was not followed anywhere and could not be reasonably considered "standard care," but was rather "unusual" and therefore experimental care (Cortes-Puch et al. 2016; Macklin and Natanson 2020). They also make useful suggestions about how to prevent future mischaracterizations of "usual care."

Another central problem with SUPPORT is denial of death, illustrated by the inability of researchers, IRBs, and supporters of SUPPORT, to acknowledge the fact that the study itself could put the newborn subjects at increased risk of death. This is understandable. It is, and should be, difficult to justify risky 